e-Journal of Educational

Research, Assessment and Evaluation

\section{REIIEVE}

Revista ELectrónica de Investigación y EValuación Educativa

\title{
PISA Y LA COMPETENCIA CIENTÍFICA: UN ANÁLISIS DE LAS PRUEBAS DE PISA EN EL ÁREA DE CIENCIAS
}

\section{[PISA and scientific competence: an analysis of the PISA tests in the Area of Science]}

$$
\text { by / por }
$$

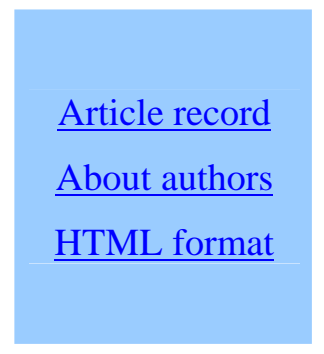

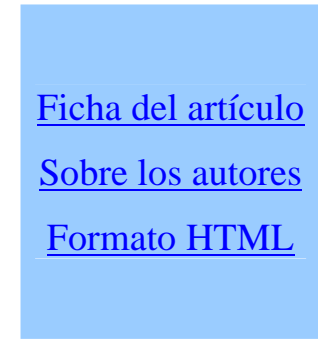

\begin{abstract}
This work is part of an investigation focusing on the educational evaluation of the skill of Learning how to Learn, which addresses the issue of basic skills that students needs to develop to function throughout life in the contemporary social context. One purpose of the study is to analyze the potential of external and diagnostics evaluations to estimate the level of acquisition of these skills. Focusing on a methodology of qualitative and quantitative analysis of the PISA tests released in the specific area of Science (2000-2006), we conclude that these tests require scientific skills of low complexity, with marked presence of mere reproduction. In addition, we appreciate the gap between the concept of competence proposed DeSeCo and what PISA really evaluates according to the results obtained in the area of Science.
\end{abstract}

\section{Keywords}

Assessment; Evaluation; Education; Skills; Competence; PISA; Sciences.

\section{Resumen}

El presente trabajo forma parte de una investigación centrada en la evaluación educativa de la competencia de Aprender cómo Aprender, en la que se aborda la temática de las competencias básicas que los estudiantes necesitan desarrollar para desenvolverse durante toda la vida en el contexto social contemporáneo. Uno de los propósitos del estudio es analizar el potencial de las evaluaciones externas y diagnósticas para estimar el grado de adquisición de estas competencias. Centrándonos en una metodología de análisis cualitativo y cuantitativo de las pruebas liberadas de PISA en el área específica de Ciencias (2000-2006), concluimos que estas pruebas demandan capacidades científicas de baja complejidad, con acentuada presencia de la mera reproducción. Además, valoramos la distancia existente entre el concepto de competencia propuesto en DeSeCo y lo que realmente parece evaluar PISA a la luz de los resultados obtenidos en el área de Ciencias.

\section{Descriptores}

Evaluación; Educación; Competencias; PISA; Ciencias.. 


\section{Introducción}

El presente trabajo forma parte de una investigación del grupo HUM-311 del Departamento de Didáctica y Organización Escolar de la Universidad de Málaga sobre la evaluación educativa de la competencia de Aprender cómo Aprender ${ }^{[1]}$. En el desarrollo de dicho proyecto, se aborda la temática de las competencias básicas como un mecanismo potencial para el desarrollo de las capacidades útiles para la vida, específicamente las competencias de orden superior que podrían agruparse bajo el constructo de "aprender cómo aprender” (Hargreaves, 2005). Uno de los propósitos del estudio es analizar el potencial de las evaluaciones externas y diagnósticas para estimar el grado de adquisición de estas competencias. Entre estas evaluaciones destaca el Programa Internacional para la Evaluación de Estudiantes (PISA), que basa su fundamentación en el concepto holístico de competencia básica establecido en el Informe DeSeCo para la OCDE (2002), que también sirvió para el establecimiento de la Recomendación del Parlamento Europeo y del Consejo de 18 de diciembre de 2006 sobre las competencias clave para el aprendizaje permanente, que, a su vez, fue recogida en la nueva reforma educativa española: la Ley Orgánica 2/2006 de Educación -LOE- (Ministerio de Educación, 2006). Desde este marco, nos proponemos el objetivo de estudiar y valorar las pruebas del Proyecto PISA de evaluación de las competencias básicas. Para este objetivo, dentro del equipo de investigación, se establecen grupos de trabajo específicos para cada área curricular, de modo que, en este artículo, se resumen las principales conclusiones del área de Ciencias, es decir, la llamada competencia científica, denominada en la LOE: “Competencia en el conocimiento y la interacción con el mundo físico".

Aunque el proyecto de evaluación PISA está removiendo los asientos de nuestro sistema educativo desde su primer informe en el año 2000, fue especialmente en el año
2006 (OCDE, 2006), dedicado a la evaluación de las Ciencias, cuando empieza a tener una repercusión destacable en este área curricular, si bien, hasta hoy, parece que ha habido más preocupación por explicar la naturaleza y fundamento de las pruebas (ej. Harlen, 2002; Oñorbe, 2008) y la lectura de los resultados y sus implicaciones (Camaño, 2007; Puente Azcutia, 2008). Las principales líneas de investigación se han suscitado no sólo a partir de los resultados obtenidos, sino también sobre la propia técnica evaluativa seguida. Así, por ejemplo, un aspecto crítico de esto último estriba en la dificultad de evaluar el conocimiento científico mediante pruebas de lápiz y papel (Psalidas et al., 2008). Otros estudios se centraron en analizar los materiales curriculares, como Hatzinikita et al. (2008), que encontraron que había una fuerte disparidad entre el tipo de tareas que plantea PISA y los libros de textos usados en los centros educativos, lo que en parte explicaría los bajos rendimientos obtenidos en las pruebas. En una línea diametralmente opuesta, destaca una larga lista de investigadores que encuentran en las pruebas de PISA una oportunidad para la mejora de las prácticas actuales de enseñanza. Así, por ejemplo, Hernández (2001) encuentra en los informes de PISA una oportunidad estimuladora para repensar la tarea que se lleva a cabo en los centros, al ponerse en evidencia que la escuela tiende a enseñar para la reproducción, mientras que las pruebas PISA están dirigidas a la aplicación o transferencia de conocimientos a situaciones cotidianas. En una línea similar, pero específicamente para el área de Ciencias, Gil y Vílchez (2001) han venido planteando en distintos foros que los Informes PISA constituyen en conjunto un instrumento potencialmente valioso para la mejora del aprendizaje, la enseñanza y los currículos. Otras investigaciones han abordado la problemática del ajuste curricular existente entre nuestro sistema educativo y el planteamiento de competencias que pretende evaluar PISA, concluyéndose que el currículum de la LOE no se ha elaborado para abordar una enseñanza del tipo que 
subyace en las competencias que evalúa PISA, existiendo áreas de la educación científica, principalmente de tipo procedimental, que no se han contemplado en la Ley.

A partir de este panorama, resulta evidente que apenas hay investigaciones que hayan abordado analíticamente el contenido de las pruebas de PISA, tratando de analizar en profundidad si realmente evalúan las competencias científicas en el sentido holístico propuesto por el informe DeSeCo de la OCDE. Éste ha sido precisamente el objetivo del trabajo que exponemos en el presente artículo, valorar si las pruebas PISA pueden ser consideradas unas herramientas útiles para evaluar competencias dentro de las estrategias generales del constructo de "aprender cómo aprender".

\section{PISA en el área de Ciencias}

Como es conocido, el modelo de evaluación comparativa de PISA se caracteriza por ser muestral (estudiantes de 15 años) y cíclico (trienal), a través de tres áreas principales de evaluación: Lectura (2000), Matemáticas (2003) y Ciencias (2006). Bajo este modelo, la prueba está diseñada para conocer las habilidades de los estudiantes para analizar y resolver problemas, tratando de ofrecer un perfil de las capacidades de los estudiantes de quince años de todos los países donde se aplica. Por otra parte, aunque PISA reconoce en sus fundamentos su pretensión de incidir en las políticas educativas, no debemos olvidar que también reconoce no estar ligado al currículum ni a planes de estudios específicos. Lo que pretende PISA, en definitiva, es evaluar 'competencias', cuya aproximación empírica en el área de Ciencias se realiza interrogando a los estudiantes sobre su capacidad para identificar cuestiones científicas, explicar fenómenos científicamente y utilizar las pruebas científicas. En este sentido, desde los fundamentos teóricos de PISA en el área de Ciencias, destaca el enfoque de evaluación en torno a la 'aplicación' del conoci- miento versus a la 'memorización de conceptos' (OCDE, 2006).

Cabe destacarse que, en el área de Ciencias, PISA muestra una aparente evolución en su fundamentación teórica desde el año 2000, en la que se hablaba de "formación científica" sin alusión al concepto de "competencia” (OCDE, 2002). Posteriormente, tras el Informe de DeSeCo (2002), en PISA2003, se empieza a abordar la noción de "competencia científica”, aunque en los mismos términos que en el año 2000, es decir, como un sumatorio de conocimientos, procesos y situaciones o contextos (personal, público y global) (OCDE, 2004). Finalmente, en el 2006, año específico de evaluación en el área de Ciencias, se introduce el concepto de "competencia científica" aplicado a un individuo concreto, manteniendo el objetivo de evaluar 'conocimiento' (conceptos) y 'aplicación del mismo' a una situación o contexto (capacidades) y añadiendo, como única novedad, la ‘disposición’ (actitud) del alumnado hacia las pruebas y el conocimiento científico (OCDE, 2006).

De este modo, PISA-2006 trata de poner de manifiesto las competencias científicas a través del dominio de los procedimientos científicos que están en la base de las preguntas, la comprensión de las capacidades que están presentes en su resolución y la valoración de las actitudes que presenta el alumnado hacia la ciencia actual. Así, PISA evalúa el conocimiento científico a través de tres dimensiones: a) Los procesos o destrezas científicas; b) Los conceptos y contenidos científicos; y c) El contexto en que se aplica el conocimiento científico. Por otra parte, PISA identifica cinco procesos científicos: Reconocer cuestiones científicamente investigables; identificar las evidencias necesarias en una investigación científica; extraer o evaluar conclusiones; comunicar conclusiones válidas; demostrar la comprensión de conceptos científicos en determinadas situaciones. Estos procesos científicos se organizan en tres grupos de competencias según el 
tipo de capacidad de pensamiento predominante que se requiere para resolver las preguntas que se presentan: a) Descripción, explicación y predicción de fenómenos científicos; b) Comprensión de la investigación científica; y c) Interpretación de evidencias y conclusiones científicas. Esta compartimentación de la competencia en áreas y capacidades, que finalmente son las que pretende evaluar PISA, supone, a nuestro juicio, un distanciamiento de la concepción holística del concepto de competencia definido por DeSeCo, siendo difícil entender que este sistema analítico evalúe la competencia científica como la define PISA: "La capacidad de emplear el conocimiento científico para identificar preguntas y extraer conclusiones basadas en hechos, con el fin de comprender $y$ poder tomar decisiones sobre el mundo natural y sobre los cambios que ha producido en él la actividad humana" (OCDE, 2006).

\section{Descripción del proceso metodológico}

Como ya hemos comentado anteriormente, este artículo nace a raíz de una investigación desarrollada por el Grupo de Investigación HUM-311 de la Universidad de Málaga, el cual se ha organizado en torno a tres subgrupos de análisis según el área evaluada: Lengua, Matemáticas y Ciencias. El subgrupo encargado del análisis del área de Ciencias, que nos ocupa en este artículo, está compuesto por cinco investigadores del área de Didáctica General (cuatro doctores y un licenciado en Pedagogía) más un investigador doctor experto en la Didáctica de las Ciencias.

La investigación de las pruebas de PISA en el área de Ciencias tiene su inicio en el año
2008 a través de la realización de un estudio preliminar en profundidad de los diferentes marcos teóricos relacionados tanto con los Informes de PISA como con investigaciones relevantes sobre el ámbito de las competencias educativas y el constructo de Aprender cómo Aprender en general. Una vez analizados estos marcos teóricos, el grupo de Ciencias decidió pasar al análisis propiamente dicho de las pruebas liberadas de PISA en dicha área, siguiendo las que aparecían en las publicaciones que se especifican a continuación: Informe Español de PISA 2006 (4 unidades), marco de la Evaluación de PISA 2006 (16 unidades) y los ítems liberados de PISA 2000 y 2003 (13 unidades).

Para el análisis de las diferentes pruebas, se utilizó una ficha en formato web, de forma que cada investigador analizaba los ítems de las pruebas PISA y éstos eran volcados a una base de datos común para su posterior análisis en SPSS (puede verse, en la figura 1, la ficha común; en la 2, el formato de datación). Estos análisis ocuparon todo el año 2009, sometiéndose continuamente a discusión para llegar a una triangulación de los resultados obtenidos en los análisis realizados por cada miembro del grupo, contrastándose además con el investigador-experto con objeto de validar los hallazgos, lo que tuvo lugar tanto mediante reuniones presenciales mantenidas para tal efecto (con una periodicidad de un mes) como a través de una Plataforma Virtual, basada en el sistema Moodle, que ha favorecido el contacto inmediato y permanente entre los seis investigadores participantes (gracias a los foros y el intercambio de información en formato electrónico fundamentalmente). 
Gallardo-Gil, Monsalud; Fernández-Navas, Manuel; Sepúlveda-Ruiz, María-Pilar; Serván, María-José; Yus, Rafael \& Barquín, Javier (2010). PISA y la competencia científica: Un análisis de las pruebas de PISA en el Área de Ciencias. RELIEVE, v. 16, n. 2, p. 1-17. http://www.uv.es/RELIEVE/v16n2/RELIEVEv16n2_6.htm

Análisis Pruebas:

1. Título:

2. $\mathrm{N}^{\circ}$ de pregunta:

3. Año:

- Ärea:

- Tema:

- Proceso:

- Competencia requerida:

4. Pautas de corrección (respuesta única o cuando plantean diversos niveles de respuesta):

- Tipo de respuesta:

$\mathrm{N}^{\circ}$ de opciones: Máxima puntuación (), puntuación parcial (), ninguna puntuación ().

5. Capacidades (respuesta con calificación máxima):

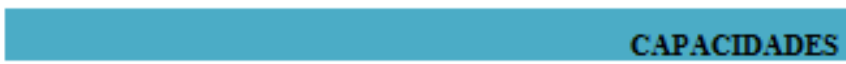

\begin{tabular}{|l|l|l|l|l|l|} 
Reproducción & Aplicación & $\begin{array}{l}\text { Comprensión/ } \\
\text { Reflexión }\end{array}$ & Transferencia & Heurística & $\begin{array}{l}\text { Comunicación/ } \\
\text { Argumentación }\end{array}$
\end{tabular}

NIVELES

6. Observaciones:

Figura 1: Ficha base de análisis 
Gallardo-Gil, Monsalud; Fernández-Navas, Manuel; Sepúlveda-Ruiz, María-Pilar; Serván, María-José; Yus, Rafael \& Barquín, Javier (2010). PISA y la competencia

científica: Un análisis de las pruebas de PISA en el Área de Ciencias. RELIEVE, v. 16, n. 2, p. 1-17. http://www.uv.es/RELIEVE/v16n2/RELIEVEv16n2 6.htm

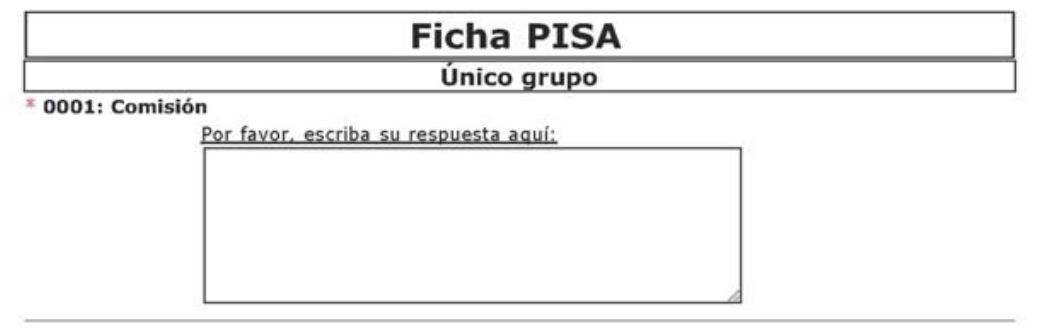

* 0002: Título de la pregunta

Por favor, escriba su respuesta aqui:

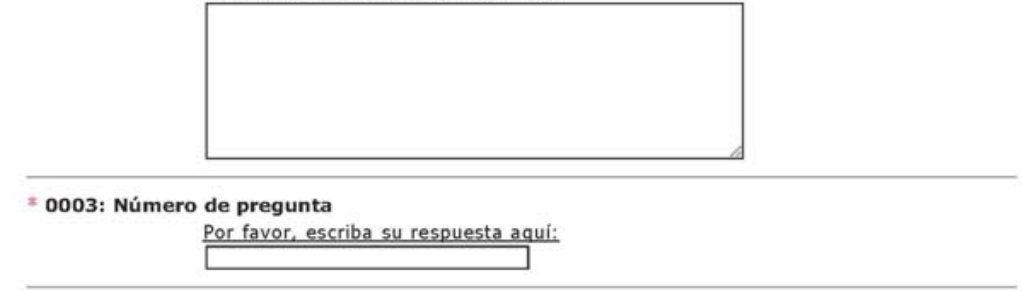

0004: Año de la prueba

Por favor, escriba su respuesta aqui:

0005: Tipo de respuesta

Marar el ípo de por favor, eliia sólo una de las siguientes entradas

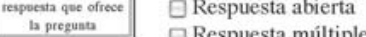

$\exists$ Respuesta múltiple

घOtro

\begin{tabular}{|c|c|}
\hline 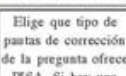 & $\begin{array}{l}\text { Por favor, elija sólo una de las siquientes entradas: } \\
\text { Puntuación única (máxima o ninguna) } \\
\text { Puntuación varia (máxima, parcial,...) }\end{array}$ \\
\hline $\begin{array}{l}\text { PSSA si hy uma } \\
\text { disica pasibilidad }\end{array}$ & Comente su opción aqui: \\
\hline 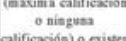 & \\
\hline 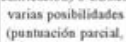 & \\
\hline 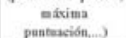 & \\
\hline
\end{tabular}

[Sólo responda esta pregunta si usted respondió 'Puntuación varia (máxima, parcial,...)' a la pregunta 0007: Número de opciones

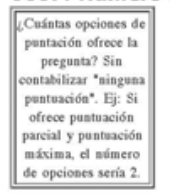

0008: Capacidades (puntuación máxima)

Por favor, elija la respuesta apropiada para cada entrada:

Reproducción

Aplicación

Comprensión / Reflexión

Transferen
Heuristica

Comunicación / Argumentación

Recuperar información

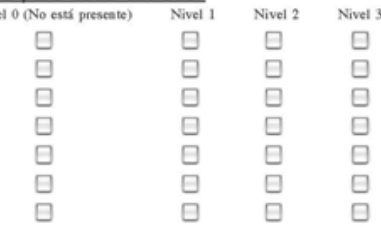

[Sólo responda esta pregunta si usted respondió 'Puntuación varia (máxima, parcial, ,...)' a la pregunta 0009: Capacidades (Opción puntuación 2)

Por favor, elija la respuesta apropiada para cada entrada:

Reproducción

Aplicación

Comprensión / Reflexión

Transferencia

Comunicación / Argumentación

Recuperar información

\begin{tabular}{|c|c|c|}
\hline & & \\
\hline$\square$ & $\theta$ & $\boxminus$ \\
\hline 日 & 日 & ㅂ \\
\hline 日 & ㅂ & 日 \\
\hline 昌 & 日 & ㅂ \\
\hline $\begin{array}{l}\text { 日 } \\
\text { 日 }\end{array}$ & 昌 & 星 \\
\hline 日 & 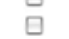 & 正 \\
\hline
\end{tabular}

[Sólo responda esta pregunta si usted respondió 'Puntuación varia (máxima, parcial,....)' a la pregunta

'0010: Capacidades (Opción puntuación 3)

Contestar s i rocede Por favor, elija la respuesta apropiada para cada entrada:

Reproducción
Aplicación
Comprensión / Reflexión
Transferencia
Heuristica
Comunicación / Argumentación
Recuperar información

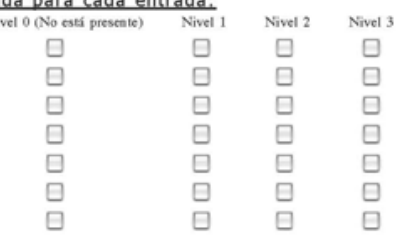

0011: observaciones

Figura 2: Formato de datación 
Tras triangular los resultados, el análisis de las pruebas se tradujo en una organización que contemplaba tanto la discusión abierta en el grupo de trabajo sobre cada prueba en particular como la "reducción" de algunas características a gradaciones numéricas. De ahí que, por un lado, se elaborara una ficha con distintos campos y, por otro, se seleccionaran una serie de capacidades que consideramos debían contemplar las pruebas a la luz de investigaciones recientes (Monereo y Pozo, 2007), identificando las siguientes capacidades en nuestros análisis:

- Reproducción: supone repetir de forma mecánica y memorística los conocimientos científicos.

- Aplicación: consiste en aplicar los conocimientos científicos aprendidos previamente a situaciones sencillas y/o conocidas.

- Comprensión/Reflexión: implica la comprensión del fenómeno científico y reflexionar sobre los conocimientos aprendidos en esta área.

- Transferencia/Conexión: supone aplicar el conocimiento aprendido a nuevas situaciones, 'conexionando' ideas, conceptos o hechos científicos.

- Heurística: la solución de la situación científica requiere el diseño de un 'plan', o la descripción de los pasos que son necesarios seguir para llegar a dicha solución.

- Comunicación/Argumentación: la prueba requiere razonar de forma argumentada para explicar el fenómeno científico que se trate, comunicando la conclusión científica a la que se llegue a través del lenguaje escrito.

Estas capacidades son demandadas por PISA en las tareas de cada ejercicio, de modo que, como cada ejercicio tiene varias preguntas o ítems que, con frecuencia, demandan más de una capacidad, es lógico que el número de capacidades detectadas sea superior al número de preguntas o ítems. Cada capacidad establecida a priori se valoraba en fun- ción primero de su presencia en la prueba y después de su peso en la misma. Así, se estableció una gradación de 1 a 3 para indicar qué capacidad/es aparecían como dominantes y cuáles eran más secundarias. Después de definir claramente estas capacidades, con objeto de comprender y analizar pormenorizadamente las pruebas, el siguiente paso fue tratar de establecer los paralelismos existentes entre éstas y las capacidades definidas por PISA. A partir de aquí, se vuelve a retomar las pruebas para profundizar en las capacidades evaluadas por PISA y su relación con las contempladas por el grupo de investigación. Bajo estas consideraciones, el grupo de trabajo volvió a someter a análisis las diferentes pruebas, con objeto de identificar claramente las capacidades definidas anteriormente, redundando así los análisis realizados y los hallazgos obtenidos, que ofrecemos de forma resumida en el siguiente epígrafe.

En síntesis, podemos decir que el proceso metodológico de nuestro estudio se ha desarrollado en las siguientes fases:

- Estudio de los diferentes marcos teóricos sobre PISA.

- Elaboración de una ficha de análisis (mostrada más arriba) en la que cada investigador introducía los análisis sobre las pruebas que le correspondía analizar: Tipo de pregunta, capacidades que demanda la prueba, grado en el que la demanda, etc.

- Una vez analizadas todas las pruebas y extraídos los datos estadísticos, así como las particularidades que cada investigador había observado en sus pruebas (lo que llamamos "incidentes críticos"), se vuelve a someter a análisis las diferentes pruebas, con objeto de redundar y validar los resultados obtenidos. Durante todo el proceso, se mantienen frecuentes reuniones presenciales y, especialmente, virtuales (a través de una Plataforma Virtual) con la finalidad de discutir los hallazgos obtenidos. 
- Como último paso, todos los frutos de la investigación, conclusiones y resultados, quedan plasmados en un completo informe sectorial del área de Ciencias, del cual es síntesis este artículo.

\section{Resultados de la investigación}

\section{Capacidades presentes en las pruebas}

A partir del análisis de las pruebas realizado por el grupo de investigación (tabla 1), encontramos que la capacidad que se requiere en más ocasiones es la que hemos definido como 'reflexión o comprensión' (un 96,08\% del total de ítems), que conlleva el entendimiento del fenómeno científico en cuestión y la reflexión sobre los conocimientos científicos implicados. En nuestro análisis, hemos detectado que esta capacidad se suele em- plear sobre los datos que se presentan en el propio ítem junto con lo que el estudiante ya conoce del fenómeno o proceso científico en cuestión, no requiriéndose, en la mayoría de las pruebas, un esfuerzo importante por parte del estudiante para llegar a su resolución.

En este sentido encontramos que, en la mayoría de los ítems, la capacidad de comprensión/reflexión no va más allá de la mera recuperación de información que aparece en el texto que se presenta al inicio del ítem a modo de estímulo, sin necesidad siquiera de que el estudiante ponga en relación la información del texto con lo que ya sabe, llegando incluso, en algunos a casos, a presentarse la información subrayada, como es el caso de la prueba que presentamos a continuación a modo de ejemplo:

\footnotetext{
Pregunta 12.3

En la frase que figura a continuación se han subrayado varlas palabras.

Los astrónomos predicen que, desde la perspectrva visual de Neptuno, podră verse un trănsito

de saturno por delante de la superficie del sol en algan momento del presente siqlo.

De las palabras subrayadas, icuăles resultarían las tres más atiles para realizar una basqueda en una biblioteca o en Internet con obleto de averiguar el momento en que se produciră ese trănsitoi
}

Figura 3: El tránsito de Venus. Ítem 12.3, del Marco teórico de 2006

Por tanto, podemos concluir que, aunque todas las pruebas implican comprensión, no todas requieren una reflexión profunda por parte del estudiante para llegar a su resolución.

Por otra parte, también encontramos que son frecuentes las preguntas de 'aplicación' (58,5\% de los ítems analizados), estando esta aplicación generalmente asociada a los contenidos que aparecen en la propia prueba. En este sentido, cabe afirmarse que las pruebas que hemos analizado, salvo en pocas ocasiones, se pueden responder de forma efectiva comprendiendo la información que se aporta en la misma actividad y aplicando los conocimientos que se suelen presentar en el propio ítem.

En otras ocasiones, hay que aplicar esta comprensión en una situación nueva, es de- cir, se requiere 'transferencia' del conocimiento, pero ésta aparece tan sólo en un 32,36\% de los ítems analizados. Del mismo modo, encontramos que otra de las capacidades superiores definidas por el grupo de investigación como la capacidad de diseñar estrategias para resolver la situación planteada, es decir, la 'heurística', aparece en un porcentaje muy bajo en las pruebas analizadas $(12,78 \%)$.

En este sentido, aunque las capacidades de transferencia y de heurística/creación aparecen con baja frecuencia en las pruebas analizadas, decidimos contemplarlas en los análisis en tanto que podemos ofrecer algún ejemplo donde están presentes, como es el caso de la prueba "Plantas de energía eólica" (Marco de la Evaluación, 16.2, 2006, p. 183). En ella, se proporcionan al estudiante una serie de condiciones que debe cumplir la 
velocidad del viento para que los aerogeneradores generen energía y se le pide que elija, de entre cuatro gráficos, cuál representa mejor las condiciones descritas. Consideramos que esta pregunta implica transferencia de conocimientos a la situación planteada, así como el diseño de un plan para elaborar la respuesta (heurística). Cuando nos enfrentamos a la pregunta, podemos decidir que primero vamos a hacer una lista de todas las variables que entran en las condiciones (V1, V2, V3, W), luego vamos a intentar dibujar la gráfica y luego vamos a comparar nuestra gráfica con las respuestas proporcionadas. La pregunta, en definitiva, no se puede contestar directamente, sino que requiere pararse a pensar cómo contestarla, qué pasos dar para encontrar la respuesta, cómo ordenar e interpretar los datos proporcionados, etc.
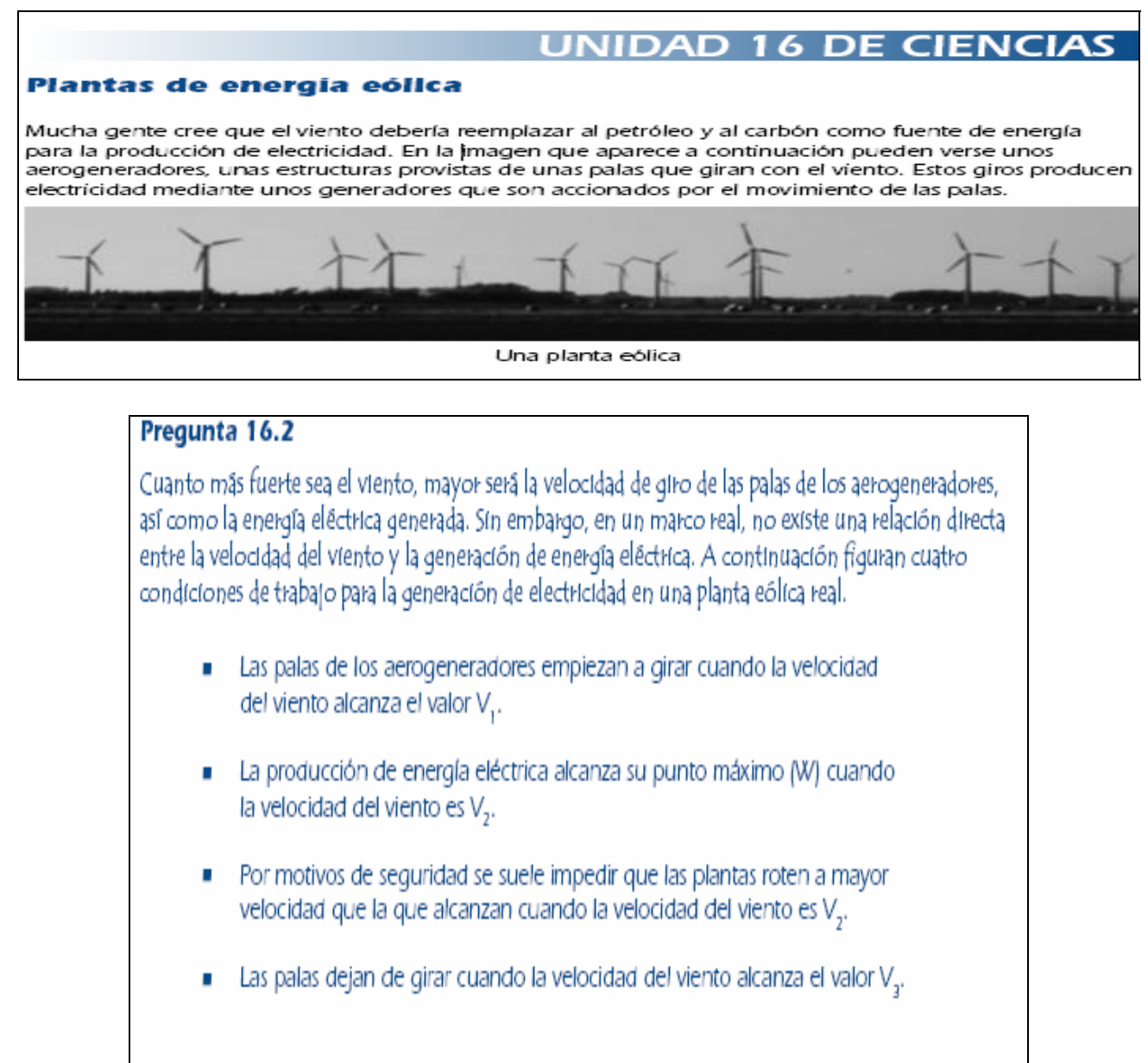

Figura 4: “Plantas de energía eólica”, del Marco de la Evaluación, 16.2, 2006, p.183

Por otra parte, aunque, como hemos indicado, no son nada despreciables la cantidad de preguntas que se pueden responder aplicando sin mayor dificultad una información ya conocida por parte del estudiante, muy pocas, sin embargo, reclaman una 'reproducción' de conocimientos científicos tal y como hemos definido esta capacidad, relacio- nada con la 'memorización mecánica'. Concretamente, aparece tan sólo en un 16,67\% de los casos, lo que podría relacionarse con la definición de 'competencia' que se defiende desde el propio programa PISA, donde la reproducción queda claramente descartada en la valoración de las competencias. 
Tabla 1. Resultados del análisis de pruebas PISA (\% Puntuación máxima)

\begin{tabular}{|l|c|c|c|c|c|c|}
\cline { 2 - 7 } \multicolumn{1}{c|}{} & RP & AP & RF & TR & HE & AR \\
\hline No presente & 67,73 & 34,31 & 1,96 & 49,02 & 72,55 & 54,90 \\
\hline 1 & 12,75 & 28,31 & 12,75 & 12,75 & 1,96 & 4,90 \\
\hline 2 & 3,92 & 12,75 & 26,47 & 17,65 & 2,94 & 9,80 \\
\hline 3 & 0,00 & 17,65 & 56,86 & 1,96 & 7,84 & 17,65 \\
\hline NR & 19,61 & 6,86 & 1,96 & 18,63 & 14,71 & 12,75 \\
\hline Presente & 16,67 & 58,5 & 96,08 & 32,36 & 12,78 & 32,35 \\
\hline
\end{tabular}

Todo parece indicar, pues, que las pruebas pretenden medir el grado de adquisición de determinadas habilidades relacionadas con la ciencia, aunque no necesariamente con ella, sino más bien con lo que podríamos llamar “pensamiento lógico", que también está presente en las Ciencias Sociales y, por supuesto, en las Matemáticas, aplicado en este caso a problemas biológicos o geológicos.

Sin embargo, en las pruebas examinadas, no siempre se estimula la argumentación, pareciendo que lo único que interesa es la respuesta 'aplicada' por parte del estudiante, el resultado final, y no tanto el proceso que lleva al alumno/a a dar una u otra respuesta. De hecho, aún considerándose la argumentación como una de las capacidades más interesantes para la evaluación de competencias en sentido complejo, tan sólo está presente en un 32,35\% de ítems analizados.

Nuestro estudio evidencia que la argumentación es una de las capacidades centrales en la evaluación de la competencia científica, en tanto que es la capacidad que pone de relieve el pensamiento complejo del estudiante. En las pruebas analizadas, cuando la 'argumentación’ está presente, suele implicar un nivel elevado de dificultad, pero sólo se suele dar como válida una sola respuesta, que es la que obtiene mayor puntuación, desconsiderándose las relaciones que ha podido establecer el estudiante o las reflexiones que haya podido realizar.

Las preguntas abiertas, donde está presente la capacidad de argumentación, exigen que el estudiante esté habituado a razonar y a comunicar sus planteamientos, alejándose, por tanto, del mero 'reconocimiento'; además, implica que el estudiante sepa leer comprensivamente y expresarse correctamente por escrito; todo ello de suma relevancia para una auténtica evaluación de competencias.

\section{¿Qué es lo que PISA intenta valorar re- almente en las pruebas?}

Aunque PISA se propone la medición de los tres tipos de capacidades relacionadas con lo que la OCDE define como "competencia científica” (identificación de cuestiones científicas, explicación científica de fenómenos y utilización de pruebas científicas), no parece prestar especial atención a los grupos de capacidades superiores o de segundo orden ('transferencia', 'heurística' y 'argumentación’), quedándose en un concepto de competencia apegado casi de forma exclusiva al 'saber' como conocimiento y descuidando las áreas del 'saber hacer' y 'saber decir' (DeSeCo) en la evaluación de la competencia científica. Así pues, aunque en general PISA se desinteresa por el conocimiento científico entendido como mera reproducción de información de forma mecánica o memorística (lo que el grupo llama “reproducción”), encontramos que se deberían potenciar más las capacidades de segundo orden indicadas, tal y como establece el propio programa PISA en sus fundamentos teóricos.

En este sentido, cabe indicarse que PISA parece decantarse por las pruebas de elección múltiple que no admiten argumentación, una capacidad esencial, a nuestro juicio, en la competencia científica. Sólo en algunas actividades se incluye alguna pregunta abierta que requiere argumentación, pero el resto del 
problema sólo requiere elección entre respuestas múltiples. Además, cuando se utilizan preguntas abiertas, los criterios de corrección de las mismas son cerrados, valorando sólo como correctas un conjunto limitado de respuestas posibles, cuya valoración debería centrarse más en su adecuada justificación científica, capacidad mucho más relevante para la evaluación de un aprendizaje competente. Asimismo, consideramos que se debería favorecer más la creatividad del estudiante y la transferencia del conocimiento. Como hemos visto, en muy pocas ocasiones, se plantean problemas abiertos que den rienda suelta a la transferencia y mucho menos a la heurística.

Por último, se echa en falta actividades que evalúen la destreza de lectura/interpretación de imágenes gráficas (ej. esquemas, dibujos y diagramas de biología, geología, etc.), así como un mayor uso de la comunicación escrita (ej. redactar un informe, una valoración, etc.), habilidades científicas tradicionalmente consideradas importantes en la enseñanza de las ciencias y ahora en el desarrollo de la competencia científica.

\section{Relevancia del contenido de las pruebas}

Respecto a la relevancia, recordemos que hace referencia al concepto de aprendizaje situado, que se considera más idóneo para potenciar el aprendizaje. Esto significa que las actividades deben conectar con los intereses o inquietudes del alumnado para permitir que se desarrolle dicho aprendizaje. Un aprendizaje competente es aquél que el alumno/a utiliza para interpretar y actuar sobre su entorno inmediato. Por lo tanto, se plantea una importante dificultad de PISA respecto a la relevancia del contenido: ¿Cómo lograr conectar con los intereses e inquietudes del alumnado mediante una prueba estandarizada que se pasa a una gran cantidad de sujetos? La única manera que existe de que el contenido sea relevante para el alumnado pasa por el conocimiento de las inquietudes e intereses del mismo, cosa imposible de tener en cuenta en una prueba de las características de PISA, en la que la propia condición de estandarización internacional hace difícil crear contextos relevantes para realidades socio-culturales a veces muy distintas. Con ello no queremos indicar que sea imposible en todos los casos, pues, por ejemplo, la utilización de pruebas basadas en sucesos o eventos de repercusión mundial, o de fuerte repercusión mediática, ofrece marcos significativos o relevantes para todos los estudiantes, dada la globalización informativa.

Así pues, aunque el contenido de los ítems es, sin duda, actualizado y sobre temas vigentes y en discusión, PISA tiende a plantearlos de una forma academicista, desperdiciando oportunidades para plantear dilemas éticos o conflictos de actualidad en el ámbito científico para centrarse en el contenido académico de los ítems (véase "Clonación”, PISA 2000-03).

Esto puede verse también en la tipología de respuestas que PISA contempla en sus cuestiones. De todos los ítems analizados, sólo un $37 \%$ permiten la posibilidad de respuestas abiertas mientras que a las respuestas cerradas y múltiples les corresponde un 63\% (siendo más predominantes, en un $41 \%$, las respuestas múltiples). De igual modo ocurre con las pautas de corrección: mientras que para el $81 \%$ de las preguntas sólo puede obtenerse el total de la puntuación asignada o cero puntos, el $19 \%$ pueden puntuar de una manera parcial. Está claro que PISA no contempla la variedad de perspectivas y el conflicto que representa la competencia en ciencias y presta más atención a la “comprobabilidad” del conocimiento académico.

PISA parte de la base de que, si el alumno/a conoce el tema en su entorno más inmediato, automáticamente pasa a ser un tema relevante para él/ella, lo cual resulta difícil de sostener, ya que todo nuestro entorno está rodeado de diferentes temáticas cercanas a nosotros y no por ello todas nos resultan igualmente relevantes o interesantes. De esta 
manera, preguntas como: "El ejercicio físico", "El efecto invernadero", "Fumar tabaco", "Clonación”, "El cambio climático" y "Ozono", podrían tener cierta relevancia por la preponderancia del tema en los medios de comunicación. Otras les pueden parecer interesantes por su temática cotidiana: "Apta para beber", "Caries dental”, "La luz de las estrellas", "Brillo de labios", "La masa del pan”, "Luz del día” o "El chocolate”. El resto de las preguntas, tales como "Lluvia ácida”, "Ultrasonido", "El catalizador”, "La biodiversidad", "La conducta del espinoso" y, específicamente, la referida a "El Gran Cañón” (de El Colorado, que nos queda un poco lejos), creemos que es más difícil que resulten relevantes para niños y niñas de quince años en general, aunque algunos y algunas de ellos y ellas pudieran tener una afición especial por la ciencia o la naturaleza que hiciese que encontrasen la pregunta relevante. Lo que está claro es que esto no pueden ser más que apreciaciones sin ningún argumento sobre una "supuesta" relevancia, ya que es imposible determinar el grado de relevancia del contenido de las pruebas.

En síntesis, encontramos que es difícil plantear preguntas relevantes para el alumnado mediante una prueba estandarizada para un abanico tan variado de realidades socioculturales debido al desconocimiento o imposibilidad de conectar con las inquietudes e intereses de tan diversa población muestreada y, por tanto, con el conocimiento necesario para desarrollar una aprendizaje situado, significativo, relevante y/o competente.

Por otro lado, cabe destacarse que el esfuerzo por parte de PISA para presentar temas actuales y con repercusión mediática en sus ítems no parece ser más que una actualización del contenido y no una nueva manera de tratarlo, lo que termina por caer, en muchas ocasiones, en la búsqueda de un grado de conocimiento, en lugar de la competencia en un ámbito, tal y como se hace tradicionalmente en nuestras escuelas.

\section{¿A qué no presta atención PISA?}

Si bien no parece, por las evidencias encontradas en la investigación, que PISA esté valorando 'competencias' tal y como se plantean en DeSeCo (una cuestión importante a pensar puede ser si esto puede hacerse mediante pruebas estandarizadas), sí podemos esbozar, tras el análisis de las pruebas, un panorama general de aspectos a los que el Programa parece prestar menor atención.

Parece evidente, viendo los porcentajes anteriores de respuestas cerradas y abiertas presentados más arriba, que la argumentación y la comunicación no son capacidades que PISA intente valorar con interés en sus pruebas (esta capacidad no aparece en el $67.65 \%$ de los ítems analizados). Esto se debe quizás a la dificultad que plantearía de cara a la corrección de las pruebas, ligado a una preocupación por la búsqueda de la objetividad en la corrección, imposible de garantizar si la argumentación y la comunicación son muy predominantes.

Asimismo, como hemos visto, en muy pocas ocasiones, plantea problemas abiertos que den rienda suelta a la capacidad de crear o a la heurística (esta capacidad no aparece en el $87.22 \%$ de los ítems analizados). Al parecer, a PISA le interesa más el seguimiento de las leyes y teorías científicas (mediante preguntas cerradas) que el pensamiento divergente implícito en el espíritu creativo, fundamental en el avance de la ciencia.

Por otra parte, una de las capacidades que reclama PISA es la de 'búsqueda de información científica y su selección’. Es una lástima que esta búsqueda se restrinja únicamente a la información que se proporciona en el enunciado. Para que el alumnado pueda desplegar al máximo su competencia (recordemos que no tiene que ver con el contenido) en cualquier aspecto, es imprescindible que tenga a su alcance todo tipo de información, incluido un portátil conectado a la red (como parece haberse propuesto en PISA 2009, con una aplicación 'cerrada'). 
Otro aspecto llamativo de las pruebas de PISA es la restricción de los problemas a temas puramente académicos, con escasa o nula conexión con temas transversales. Así, aunque algunas actividades plantean cuestiones de salud y medio ambiente (nunca sobre otros derechos humanos), no aparecen preguntas en las que aflore en profundidad la actitud del alumno/a; asimismo, tampoco reclaman reflexiones o dilemas éticos por parte del estudiante, cuestiones éstas que se supone están indefectiblemente ligadas a la actividad científica.

Relacionado con esto, llama también la atención la presencia de algunos ítems que se plantean únicamente para intentar valorar la actitud del alumno/a frente a las ciencias, aunque de un modo superficial. Curiosamente, no aparecen liberadas las pautas de corrección para dichos ítems, con lo cual entendemos que no cuentan para la puntuación de la prueba (véase pregunta 1.6 de "Apta para beber” del Marco de 2006).

Por otra parte, en relación con el contenido, podría plantearse quizás un posible sesgo cultural en la elección de los temas propuestos, propios de una clase social determinada, alejados de culturas locales y minoritarias (véase "Cirugía con anestesia general" del Marco de 2006, "El cambio climático" de PISA 2000-03 o "El catalizador" del Marco de 2006).

La preocupación de los diseñadores de las pruebas por conseguir evitar cualquier sesgo tanto en el contenido de la pregunta como en la exactitud de las respuestas provoca que se eviten en las pruebas contenidos o temas problemáticos, huyendo de la "contaminación” de las opiniones. La ciencia, en la orientación más positivista, ha de respetar determinados procesos y los hallazgos obtenidos no admiten respuestas equívocas. Ese conocimiento exacto y asimilación de sus métodos puede generar un racionalismo exacerbado y una actitud/creencia hacia el conocimiento científico como un saber acrítico y falto de referentes históricos, sociales, etc., que expliquen los porqués de la deriva de la ciencia hacia focos de investigación determinados.

No se han observado en las pruebas temas de conocimiento sujetos al debate, que pudieran sembrar la duda en los estudiantes por las opciones a elegir o las respuestas requeridas. Ahora bien, ese alejamiento de temas 'problemáticos' presenta a la ciencia y al conocimiento científico como una metáfora de la verdad suprema que, para algunos, es la nueva religión. De ahí que, en la tipología de las pruebas, parece existir la estrategia de los evaluadores de eliminar el "ruido de fondo" en la medición, es decir, eliminar cualquier cosa que no sea un procedimiento científico estricto. Esto es coherente con la forma en que se compartimenta el conocimiento en todos los currículos educativos de la OCDE.

La inclusión de la dimensión "actitudes" en 2006 indica un avance en ámbitos como la salud y el medio ambiente, pero nada aparece relacionado con el juicio moral sobre la actividad científica.

En las pruebas analizadas, pues, parece haber una falta total de atención a las implicaciones sociales y/o morales de las cuestiones tratadas, por ejemplo, en aspectos relacionados con la salud, el medio ambiente o la investigación científica. En general, estas ausencias podemos encontrarlas en pruebas como: "El Gran Cañón”, "Fumar Tabaco”, "El ejercicio físico”, “Ozono”, "El cambio climático”, "El efecto invernadero”, "Clonación”. En las citadas pruebas, no se hace alusión a los impactos del turismo en el medio ambiente, los intereses de las multinacionales sobre determinadas enfermedades, la ética sobre la reproducción, etc., soslayando, como decimos, aquellos apartados que son causa de debates morales, políticos, ecológicos, etc. 


\section{Conclusiones}

Como hemos analizado, PISA pretende establecer un marco común, internacional, de evaluación del rendimiento de los estudiantes de quince años, entendido como nivel de competencia. Bajo el concepto de 'competencia', PISA ha tratado de promover la valoración de la 'utilidad' y 'aplicación' del conocimiento sobre la reproducción en la formación de la competencia, analizando y especificando los conocimientos, capacidades y actitudes que intervienen en la formación de la competencia científica. Sin embargo, en nuestro análisis, encontramos bastantes limitaciones de cara la evaluación de la competencia científica en los términos que define el propio programa PISA -como un 'saber hacer complejo'-. En este sentido, consideramos que se debería potenciar una aproximación más holística e interdisciplinar a las competencias, en tanto que encontramos una falta de correspondencia entre el concepto de competencia que plantea DeSeCo y la forma en que PISA las evalúa finalmente, fragmentándolas en disciplinas, capacidades, habilidades y destrezas; alejándose de las capacidades de orden superior como son la transferencia, la heurística y la argumentación; haciendo, en definitiva, que la competencia pierda su sentido. Es posible que la pretensión de someter a estas pruebas a un análisis estadístico convencional obligue a los diseñadores a demandar ejercicios de respuestas cerradas para facilitar la corrección de los mismos y evitar el componente de subjetividad que conlleva una valoración.

Por otra parte, consideramos que se debería aprovechar todo el potencial crítico de las preguntas, en tanto que un artificio llamativo de las pruebas de PISA parece ser la restricción de los problemas a temas puramente académicos, con escasa o nula conexión con los temas transversales y de la 'vida real'. Es cierto que algunas actividades plantean cuestiones de salud y medio ambiente, pero no profundizan en ellas, no plantean preguntas que revelen en profundidad la actitud del alumno/a, ni plantean reflexiones o dilemas éticos ligados a la actividad científica.

En otro orden de cosas, consideramos que permitir el acceso a material de consulta para responder las preguntas debe ser un recurso imprescindible para la evaluación de competencias; el alumnado, desde este punto de vista, debería tener a su alcance todo tipo de información.

A modo de conclusión final, cabría preguntarse para futuras investigaciones si este modelo de evaluación de competencias que plantea PISA (mediante pruebas estandarizadas) es o debe ser el más adecuado para las finalidades que se proponen desde el propio Programa de evaluación. Algunas de las dificultades que hemos visto a lo largo de todo el artículo, podrían resultar insalvables para una prueba de estas características: la relevancia del contenido de las pruebas, la corrección objetiva de preguntas que pudieran plantear argumentación y/o heurística, etc. Por lo tanto, estamos en condiciones de esgrimir la necesidad de otros modelos más acordes para la evaluación de competencias educativas en sentido holístico, poniendo en duda, por tanto, si la solución a la evaluación de competencias está en el perfeccionamiento de la prueba PISA o en el modelo elegido para la evaluación de las mismas. Tal vez los países implicados debieran acudir a enfoques cualitativos que permitieran la observación del comportamiento de los adolescentes en situaciones naturales para matizar los resultados estadísticos globales, puesto que en el horizonte no se atisba una desaparición de estos análisis mundiales.

\section{Bibliografía}

Camaño, A. (2007). La evaluación PISA en Ciencias en 2006 en España e Iberoamérica. Alambique. Did. Cien. Exper. 47, 5-11.

Gil Pérez, D. y Vilches, A. (2006). ¿Cómo puede contribuir el proyecto PISA a la mejora de la enseñanza de las Ciencias (y de 
otras áreas de conocimiento)?. Revista de Educación, No Extra. 295-331.

Hargreaves, D.H. (2005). Personalising learning 3: learning to learn \& the new technologies. London: Specialist Schools Trust.

Harlen, W. (2002). Evaluar la alfabetización científica En el programa de la OCDE para la evaluación internacional de estudiantes (PISA). Enseñanza de las Ciencias, 20 (2), 209-216.

Hatzinikita, V.; Dimopoulos, K. \& Christidou, K. (2008). PISA test items and school textbooks related to science: A textual comparison. Science Education, 92 (4), 664 687.

Hernández, F. (2006). El informe PISA: una oportunidad para replantear el sentido del aprender en la escuela secundaria. Revista de Educación, extraordinario PISA, 357379.

Ministerio de Educación (2006). Ley Orgánica 2/2006, de 3 de mayo, de Educación. Boletín Oficial del Estado no 196 (4 de mayo), 17158-17207.

Monereo, C. y Pozo, J.I. (2007). Competencias para (con) vivir con el siglo XXI. Cuadernos de Pedagogía, 370, 12-18.

Organización para la Cooperación y el Desarrollo Económico (OCDE). (2002). Conocimientos y aptitudes para la vida. Primeros resultados del PISA 2000 de la OCDE. Madrid: Santillana-MEC.

Organización para la Cooperación y el Desarrollo Económico (OCDE). (2002). La definición y selección de competencias clave (DeSeCo). Resumen ejecutivo. www.OECD.org/edu/statistics/deseco (www.deseco.admin.ch) (consultado el 10 diciembre 2009).

Organización para la Cooperación y el Desarrollo Económico (OCDE). (2004). Marcos teóricos de PISA 2003. Conocimientos y destrezas en Matemáticas, Lectura, Ciencias y Solución de problemas, Madrid: Santillana-MEC.

Organización para la Cooperación y el Desarrollo Económico (OCDE). (2006). PISA 2006. Marco de la evaluación. Conocimientos y habilidades en Ciencias, Matemáticas y Lectura. Madrid: Santillana-MEC.

Oñorbe, A. (2008). Las pruebas de evaluación en Ciencias del proyecto PISA. Alambique. Did. Cien. Exper. 57, 41-52.

Psalidas, A., Apostolopoulos, C. \& Hatzinikita, V. (2008). Investigating factors affecting students' performance to PISA Science items. Journal of Engineering Science and Technology Review 1, 90-97.

Puente Azcutia, J. (2008). PISA 2006: resultados españoles en Ciencias. Alambique. Did. Cien. Exper. 57, 12-22.

\section{Notas}

[1] Proyecto I+D (ref. SEJ-2007-66967), financiado por el Ministerio de Educación y dirigido por el profesor D. Ángel I. Pérez Gómez (Universidad de Málaga). 


\section{ABOUT THE AUTHORS / SOBRE LOS AUTORES}

Gallardo-Gil, Monsalud (monsalud@uma.es). Doctora en Ciencias de la Educación por la Universidad de Málaga. Es la autora de contacto para este artículo. Pertenece al grupo de investigación "Innovación y Evaluación Educativa en Andalucía” (HUM-0311). Sus líneas principales de investigación son: formación del profesorado, TIC, evaluación educativa y procesos de enseñanza-aprendizaje en el contexto rural. Buscar otros artículos de esta autora en Google Académico /Find other articles by this author in Scholar Google

\section{Google}

Fernández-Navas, Manuel (mfernandez1@uma.es). Investigador de la Universidad de Málaga. Es Maestro de Audición y Lenguaje y Licenciado en Psicopedagogía. Pertenece al grupo de investigación “Innovación y Evaluación Educativa en Andalucía” (HUM-0311). Sus líneas de investigación son enseñanza virtual, investigación-acción, nuevas tecnologías en educación y evaluación educativa. Actualmente está trabajando en su tesis sobre enseñanza virtual en el ámbito universitario. Buscar otros artículos de este autor en Google Académico / Find other articles by this author in Scholar Google Google

Sepúlveda-Ruiz, María-Pilar (mdsepulveda@uma.es). Licenciada en Pedagogía y Doctora por la Universidad de Málaga, es Profesora titular del Dpto. de Didáctica y Organización Escolar de dicha Universidad. Pertenece al grupo de investigación "Innovación y Evaluación Educativa en Andalucía" (HUM-0311). Sus publicaciones se han centrado en la formación inicial del profesorado, principal preocupación en su trayectoria. Buscar otros artículos de esta autora en Google Académico / Find other articles by this author in Scholar Google GoOgle

Serván, María-José (servan@uma.es). Doctora en Ciencias de la Educación, es Profesora Ayudante del Departamento de Didáctica y Organización Escolar de la Universidad de Málaga. Pertenece al grupo de investigación "Innovación y Evaluación Educativa en Andalucía” (HUM-0311). Destacan sus publicaciones en torno a formación del profesorado y evaluación educativa. Buscar otros artículos de esta autora en Google Académico / Find other articles by this author in Scholar Google

Yus, Rafael (rafayus@telefonica.net). Doctor en Ciencias, es Catedrático de Ciencias Naturales y autor de varias decenas de libros y centenares de artículos de ciencia y pedagogía. Pertenece al grupo de investigación “Innovación y Evaluación Educativa en Andalucía” (HUM-0311). Ha venido centrando su labor en didáctica de las ciencias, temas transversales, educación en valores y currículum integrado. Buscar otros artículos de este autor en Google Académico / Find other articles by this author in Scho$\underline{\text { lar Google }}$ Google

Barquín, Javier (barquin@uma.es). Doctor en Ciencias de la Educación y profesor Titular de Dpto. de Didáctica y Organización Escolar de la Universidad de Málaga. Pertenece al grupo de investigación “Innovación y Evaluación Educativa en Andalucía” (HUM-0311). Trabaja en el ámbito universitario desde 1984, tratando temas de profesorado y uso de las TIC. Buscar otros artículos de este autor en Google Académico / Find other articles by this author in Scholar Google

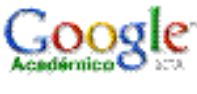


Gallardo-Gil, Monsalud; Fernández-Navas, Manuel; Sepúlveda-Ruiz, María-Pilar; Serván, María-José; Yus, Rafael \& Barquín, Javier (2010). PISA y la competencia científica: Un análisis de las pruebas de PISA en el Área de Ciencias.

RELIEVE, v. 16, n. 2, p. 1-17. http://www.uv.es/RELIEVE/v16n2/RELIEVEv16n2_6.htm

\section{ARTICLE RECORD / FICHA DEL ARTÍCULO}

\begin{tabular}{|c|c|}
\hline $\begin{array}{l}\text { Reference / } \\
\text { Referencia }\end{array}$ & $\begin{array}{l}\text { Gallardo-Gil, Monsalud; Fernández-Navas, Manuel; Sepúlveda-Ruiz, María-Pilar; Serván, María-José; } \\
\text { Yus, Rafael \& Barquín, Javier (2010). PISA y la competencia científica: Un análisis de las pruebas de PI- } \\
\text { SA en el Área de Ciencias. RELIEVE, v. 16, n. 2. } \\
\text { ttp://www.uv.es/RELIEVE/v16n2/RELIEVEv16n2 6.htm. }\end{array}$ \\
\hline Title / Título & $\begin{array}{l}\text { PISA y la competencia científica: Un análisis de las pruebas de PISA en el Área de Ciencias. [PISA and } \\
\text { scientific competence: An analysis of the PISA tests in the Area of Science]. }\end{array}$ \\
\hline $\begin{array}{l}\text { Authors / } \\
\text { Autores }\end{array}$ & $\begin{array}{l}\text { Gallardo-Gil, Monsalud; Fernández-Navas, Manuel; Sepúlveda-Ruiz, María-Pilar; Serván, María-José; Yus, } \\
\text { Rafael \& Barquín, Javier }\end{array}$ \\
\hline $\begin{array}{l}\text { Review / } \\
\text { Revista }\end{array}$ & $\begin{array}{l}\text { RELIEVE (Revista ELectrónica de Investigación y EValuación Educativa / E-Journal of Educational Re- } \\
\text { search, Assessment and Evaluation), v. 16, n. } 2 .\end{array}$ \\
\hline ISSN & 1134-4032 \\
\hline $\begin{array}{l}\text { Publication } \\
\text { date / } \\
\text { Fecha de } \\
\text { publicación }\end{array}$ & $\begin{array}{l}2010 \text { (Reception Date: } 2010 \text { June 14; Approval Date: } 2010 \text { December 26; Publication Date: } 2010 \text { De- } \\
\text { cember 26). }\end{array}$ \\
\hline & $\begin{array}{l}\text { This work is part of an investigation focusing on the educational evaluation of the skill of Learning how to } \\
\text { Learn, which addresses the issue of basic skills that students needs to develop to function throughout life in } \\
\text { the contemporary social context. One purpose of the study is to analyze the potential of external and diag- } \\
\text { nostics evaluations to estimate the level of acquisition of these skills. Focusing on a methodology of quali- } \\
\text { tative and quantitative analysis of the PISA tests released in the specific area of Science (2000-2006), we } \\
\text { conclude that these tests require scientific skills of low complexity, with marked presence of mere repro- } \\
\text { duction. In addition, we appreciate the gap between the concept of competence proposed DeSeCo and what } \\
\text { PISA really evaluates according to the results obtained in the area of Science. }\end{array}$ \\
\hline $\begin{array}{l}\text { Abstract / } \\
\text { Resumen }\end{array}$ & $\begin{array}{l}\text { El presente trabajo forma parte de una investigación centrada en la evaluación educativa de la competencia } \\
\text { de Aprender cómo Aprender, en la que se aborda la temática de las competencias básicas que los estudian- } \\
\text { tes necesitan desarrollar para desenvolverse durante toda la vida en el contexto social contemporáneo. Uno } \\
\text { de los propósitos del estudio es analizar el potencial de las evaluaciones externas y diagnósticas para esti- } \\
\text { mar el grado de adquisición de estas competencias. Centrándonos en una metodología de análisis cualitati- } \\
\text { vo y cuantitativo de las pruebas liberadas de PISA en el área específica de Ciencias (2000-2006), conclui- } \\
\text { mos que estas pruebas demandan capacidades científicas de baja complejidad, con acentuada presencia de } \\
\text { la mera reproducción. Además, valoramos la distancia existente entre el concepto de competencia propues- } \\
\text { to en DeSeCo y lo que realmente parece evaluar PISA a la luz de los resultados obtenidos en el área de } \\
\text { Ciencias. }\end{array}$ \\
\hline $\begin{array}{l}\text { Keywords / } \\
\text { Descriptores }\end{array}$ & $\begin{array}{l}\text { Assessment; Evaluation; Education; Skills; Competence; PISA; Sciences. } \\
\text { Evaluación; Educación; Competencias; PISA; Ciencias. }\end{array}$ \\
\hline $\begin{array}{l}\text { Institution / } \\
\text { Institución }\end{array}$ & Departamento de Didáctica y Organización Escolar. Universidad de Málaga. (España). \\
\hline $\begin{array}{l}\text { Publication site } \\
\text { / Dirección }\end{array}$ & http://www.uv.es/RELIEVE \\
\hline $\begin{array}{l}\text { Language / } \\
\text { Idioma }\end{array}$ & Español (Title, abstract and keywords in English) \\
\hline
\end{tabular}

\section{RELIEVE}

Revista ELectrónica de Investigación y EValuación Educativa E-Journal of Educational Research, Assessment and Evaluation

[ISSN: 1134-4032]

(C) Copyright, RELIEVE. Reproduction and distribution of this articles it is authorized if the content is no modified and their origin is indicated (RELIEVE Journal, volume, number and electronic address of the document).

(C) Copyright, RELIEVE. Se autoriza la reproducción y distribución de este artículo siempre que no se modifique el contenido y se indique su origen (RELIEVE, volumen, número y dirección electrónica del documento). 